\title{
Self-Organized Motion in a Class of Anisotropic Swarms: Convergence vs Oscillation
}

\author{
Tianguang Chu, Long Wang, and Tongwen Chen
}

\begin{abstract}
We study an anisotropic swarm model with a class of attraction and repulsion functions. It is shown that the members of a reciprocal swarm will aggregate and eventually form a cohesive cluster of finite size around the swarm center within finite time. Under mild conditions on attraction and repulsion functions, the swarm is also completely stable, i.e., every motion converges to the equilibrium point set of the system. These results are further extended to a class of nonreciprocal swarms under the detailed balance condition on coupling weights. We also present numerical simulations demonstrating complex oscillatory behavior in general nonreciprocal swarms. These results provide more insight into the effect of the interaction pattern on collective behavior of swarms.
\end{abstract}

Index Terms - Biological systems; Multi-agent systems; Pattern formation; Stability; Swarms

\section{INTRODUCTION}

Self-organization and pattern formation in swarms of biological entities are ubiquitous in nature. Examples include animal aggregation, traffic patterns, and cell migration [1], [2]. Understanding the mechanisms and operational principles of such self-organized motions in swarms can provide useful ideas for developing distributed cooperative control, formation control, coordination, and learning strategies for autonomous multi-agent systems such as autonomous multi-robot teams and autonomous air vehicles. This has motivated an increasing interest in investigating swarming behavior and its applications in recent years, and a variety of efforts have been devoted to modeling and exploring the collective dynamics in swarms [3]-[32]. The general understanding now is that the swarming behavior is a result of an interplay between a long range attraction and a short range repulsion between the individuals. Based on this, several swarm models have been proposed in the literature (see, e.g., [1], [19], [35]-[40], and the references therein).

In a recent paper, Gazi and Passino [19] proposed a simple swarm model that consists of a number of individuals (or members) with the same attraction-repulsion rule and with identical interaction strength among the individuals. They showed that the model can capture the basic features of aggregation, cohesion, and stability of a swarm. Such

This work is supported by NSFC (No. 10372002, and No. 60274001), the National Key Basic Research and Development Programme (No. 2002CB312200), and NSERC.

T. Chu and L. Wang are with the Center for Systems and Control, Department of Mechanics and Engineering Science, Peking University, Beijing 100871, China. chutg@pku . edu . cn

T. Chen is with the Department of Electrical and Computer Engineering, University of Alberta, Edmonton, Alberta T6G 2V4, Canada. properties are of interest in formation control and distributed optimization problems. However, the identical coupling strength assumption made in [19] represents only a special case in reality. In many cases interaction strength between individuals in a swarm system may vary from one pair to another, depending on relative locations of the individuals or other factors, such as different communication efficiency or bandwidth etc. This motivates our interest in investigating more general anisotropic swarms.

The purpose of this paper is to investigate collective behavior in a class of anisotropic swarms. In the next section we propose an anisotropic swarm model which includes the isotropic model of [19] as a special case. We consider two cases about the connectivity of the swarms, i.e., reciprocal and nonreciprocal interconnection topology. We show in Section 3 that the reciprocal model can also exhibit aggregation and complete stability behavior as those in [19]. The results are further extended to a class of nonreciprocal swarms in Section 4, under the detailed balance condition. For general case of nonreciprocal swarms, we give numerical simulations to illustrate that complex oscillatory motion may occur. Finally, we conclude this paper in Section 5.

The main difference of our approach from that of [19] is that, due to the anisotropy of the interaction pattern, our study on aggregation behavior relies crucially on discussion of the eigenstructure of connection matrices. The results turn out to be related to the second largest eigenvalue of the connection matrix. This provides more insight into the effect of interaction pattern on swarming behavior.

\section{THE SWARM MODEL}

We consider a swarm of $m$ individuals (members) in an $n$-dimensional Euclidean space,

$$
\dot{\mathbf{x}}^{i}=\sum_{j=1}^{m} w_{i j} \mathbf{f}\left(\mathbf{x}^{i}-\mathbf{x}^{j}\right), i=1, \ldots, m
$$

where $\mathbf{x}^{i} \in \mathbb{R}^{n}$ represents the position of individual $i, W=$ $\left[w_{i j}\right] \in \mathbb{R}^{n \times n}$ is the the interactive efficiency (or coupling weight) matrix with $w_{i j} \geq 0$ and $w_{i i}=0$ for all $i, j, \mathbf{f}(\cdot)$ is the social potential function that governs the interindividual interactions and is assumed to have a long range attraction and short range repulsion nature.

Following [20], we make the following assumptions on the social potential function: 
Assumption 1. The attraction-repulsion function $\mathbf{f}(\cdot)$ is of the form

$$
\mathbf{f}(\mathbf{y})=-\mathbf{y}\left[f_{a}(\|\mathbf{y}\|)-f_{r}(\|\mathbf{y}\|)\right], \mathbf{y} \in \mathbb{R}^{n},
$$

where $f_{a}: \mathbb{R}_{+} \rightarrow \mathbb{R}_{+}$represents (the magnitude of) attraction term and has a long range, whereas $f_{r}: \mathbb{R}_{+} \rightarrow \mathbb{R}_{+}$represents (the magnitude of) repulsion term and has a short range, and $\mathbb{R}_{+}$stands for the set of nonnegative real numbers, $\|\mathbf{y}\|=\sqrt{\mathbf{y}^{T} \mathbf{y}}$ is the Euclidean norm.

Assumption 2. There are positive constants $a, b$ such that for any $\mathbf{y} \in \mathbb{R}^{n}$,

$$
f_{a}(\|\mathbf{y}\|)=a \text { and } f_{r}(\|\mathbf{y}\|) \leq \frac{b}{\|\mathbf{y}\|} .
$$

That is, we assume a linear attraction and a bounded repulsion.

Assumption 3. There exist functions $p_{a}, p_{r}: \mathbb{R}_{+} \rightarrow \mathbb{R}_{+}$ such that for any $\mathbf{y} \in \mathbb{R}^{n}$,

$$
\nabla_{\mathbf{y}} p_{a}(\|\mathbf{y}\|)=\mathbf{y} f_{a}(\|\mathbf{y}\|), \quad \nabla_{\mathbf{y}} p_{r}(\|\mathbf{y}\|)=\mathbf{y} f_{r}(\|\mathbf{y}\|) .
$$

In other words, we assume that attraction and repulsion among the swarm members are governed by potential fields $p_{a}(\|\mathbf{y}\|)$ and $p_{r}(\|\mathbf{y}\|)$. In this case the motion of the individuals toward each other is along the gradient of these fields.

An example of the attraction-repulsion function that satisfies the above assumptions is of the form (see [19]):

$$
\mathbf{f}(\mathbf{y})=-\mathbf{y}\left[a-b \exp \left(-\frac{\|\mathbf{y}\|^{2}}{c}\right)\right]
$$

with $p_{a}(\|\mathbf{y}\|)=a>0$ and $p_{r}(\|\mathbf{y}\|)=b \exp \left(-\|\mathbf{y}\|^{2} / c\right)$. For other forms of attraction-repulsion functions, see [20].

Remark 1. The swarm model studied in [19] is a special case of model (1) with each $w_{i j}=1$, which is an isotropic system with identical interindividual interactions. By contrast, the present model in (1) is in general an anisotropic system.

We assume throughout the paper that the coupling matrix is irreducible, which implies that the swarm is fully connected and contains no isolated clusters.

In what follows, we will present results concerning the average motion, aggregation and cohesiveness, and complete stability of the swarm in (1).

\section{RECIPROCAL SWARMS}

This section considers a class of swarms in which the interactions between two individuals are reciprocal. To be specific, we make the following assumption.

Assumption 4: The swarm described in (1) is reciprocal, i.e., the coupling weights satisfy $w_{i j}=w_{j i}$ for all $i, j$.

Since a swarm system often consists of a large number of individuals that may evolve in different manners, it is usually of interest to investigate collective behavior of the system rather than to ascertain detailed behavior of each individual-which may be very difficult or even impossible in general due to complex interactions among the large number of individuals. As a first step to this goal, it is simple and convenient to study the average motion of the swam members. To do this, we define the center of the swarm as below.

Definition 1: The center of the swarm members is $\overline{\mathbf{x}}=$ $\left(\sum_{i=1}^{m} \mathbf{x}^{i}\right) / m$.

Because of the symmetry of $\mathbf{f}(\cdot)$ with respect to the origin, the reciprocity of swarm (1) implies that any two members have between them interactions with the same magnitude but in opposite directions. Hence the center of a reciprocal swarm (1) is stationary for all $t$. This fact is expressed formally in the following theorem.

Theorem 1: Under Assumptions 1 and 4, the center $\overline{\mathbf{x}}$ of the swarm described in (1) is stationary for all $t$.

Proof: It is clear by definition of the function $\mathbf{f}(\cdot)$ that $\mathbf{f}(-\mathbf{r})=-\mathbf{f}(\mathbf{r}) \forall \mathbf{r} \in \mathbb{R}^{n}$. Therefore, $w_{i j} \mathbf{f}\left(\mathbf{x}^{i}-\mathbf{x}^{j}\right)=$ $-w_{j i} \mathbf{f}\left(\mathbf{x}^{j}-\mathbf{x}^{i}\right)$. From this it is straightforward to calculate that

$$
\frac{d}{d t} \overline{\mathbf{x}}=\frac{1}{m} \sum_{i=1}^{m} \sum_{j=1}^{m} w_{i j} \mathbf{f}\left(\mathbf{x}^{i}-\mathbf{x}^{j}\right)=0,
$$

which yields the desired result.

This result says that the swarm described by (1) and (2) is not drifting on average. Note, however, that the swarm members may still have relative motions with respect to the center while the center itself stays stationary. An interesting issue is whether the swarm members will move toward the swarm center and form a cohesive cluster around it. This is dealt with in the following theorem.

Theorem 2: Under Assumptions 1, 2, and 4, all the members of the swarm described in (1) will eventually enter into and remain in the bounded region

$$
\Omega_{\rho}=\left\{\mathbf{x}: \sum_{i=1}^{m}\left\|\mathbf{x}^{i}-\overline{\mathbf{x}}\right\|^{2} \leq \rho^{2}\right\},
$$

where $\rho=(b|||W|||) /\left(a \lambda_{2}\right), \||| W|| \mid=\sum_{i, j=1}^{m} w_{i j}$, and $\lambda_{2}$ is the second smallest real eigenvalue of the matrix $H=\left[h_{i j}\right]$ with

$$
h_{i j}=\left\{\begin{array}{ll}
-w_{i j}, & i \neq j \\
\sum_{j=1, j \neq i}^{m} w_{i j}, & i=j
\end{array} .\right.
$$

Moreover, for an arbitrary small $\varepsilon>0$, the convergence of the swarm to $\Omega_{\rho+\varepsilon}$ (i.e., the $\varepsilon$-neighborhood of $\Omega_{\rho}$, defined as in (6) with radius $\rho+\varepsilon$ ) from an initial state $\mathbf{x}(0)$ outside the set will occur in finite time bounded by

$$
\tau \leq \frac{1}{a \lambda_{2}} \ln \left(\frac{\rho_{0}-\rho}{\varepsilon}\right)
$$

where $\rho_{0}=\sqrt{\sum_{i=1}^{m}\left\|\mathbf{x}^{i}(0)-\overline{\mathbf{x}}\right\|^{2}}>\rho+\varepsilon$.

Proof: Let $\mathbf{e}^{i}=\mathbf{x}^{i}-\overline{\mathbf{x}}$. By definition of $\overline{\mathbf{x}}$, we have

$$
\dot{\mathbf{e}}^{i}=-\sum_{j=1}^{m} w_{i j}\left(\mathbf{e}^{i}-\mathbf{e}^{j}\right)\left[f_{a}\left(\left\|\mathbf{e}^{i}-\mathbf{e}^{j}\right\|\right)-f_{r}\left(\left\|\mathbf{e}^{i}-\mathbf{e}^{j}\right\|\right)\right] .
$$

To estimate $\mathbf{e}^{i}$, we take the Lyapunov function for the swarm as $V(\mathbf{e})=\sum_{i=1}^{m} V_{i}\left(\mathbf{e}^{i}\right)$ where $\mathbf{e}=\left[\mathbf{e}^{1 T}, \ldots, \mathbf{e}^{m T}\right]^{T}$ and

$$
V_{i}\left(\mathbf{e}^{i}\right)=\frac{1}{2} \mathbf{e}^{i T} \mathbf{e}^{i}
$$


Evaluating its time derivative along solutions of (9) and making use of Assumption 2 and the fact that $\left\|\mathbf{e}^{i}\right\| \leq$ $\sqrt{2 V(\mathbf{e})}$ for all $i$, we have

$$
\begin{aligned}
\dot{V}(\mathbf{x})= & -\sum_{i=1}^{m} \sum_{j=1}^{m} w_{i j} \mathbf{e}^{i T}\left(\mathbf{e}^{i}-\mathbf{e}^{j}\right)\left[f_{a}\left(\left\|\mathbf{e}^{i}-\mathbf{e}^{j}\right\|\right)\right. \\
& \left.-f_{r}\left(\left\|\mathbf{e}^{i}-\mathbf{e}^{j}\right\|\right)\right] \\
\leq & -a \sum_{i=1}^{m} \sum_{j=1}^{m} w_{i j} \mathbf{e}^{i T}\left(\mathbf{e}^{i}-\mathbf{e}^{j}\right)+b \sum_{i=1}^{m} \sum_{j=1}^{m} w_{i j}\left\|\mathbf{e}^{i}\right\| \\
\leq & -a \mathbf{e}^{T}(H \otimes I) \mathbf{e}+\sqrt{2} b\|W\| V^{\frac{1}{2}}(\mathbf{e}),
\end{aligned}
$$

where $H \otimes I$ is the Kronecker product of $H$ and $I$ with $H$ as defined in (7) and $I$ the identity matrix of order $n$. To get further estimate of $\dot{V}(\mathbf{e})$, we next examine the term $\mathbf{e}^{T}(H \otimes I) \mathbf{e}$.

It is easy to see that $\lambda=0$ is an eigenvalue of $H$ and $\mathbf{u}=[l, \ldots, l]^{T}$ with $l \neq 0$ is the associated eigenvector. Moreover, since $H$ is symmetric and $h_{i j} \leq 0$ for $i \neq j$, by the irreducibility of $W$ (hence $H$ ), it follows from nonnegative matrix theory [41] that all the rest eigenvalues of $-H$ are real and negative. Therefore, we can order the eigenvalues of $H$ as $0=\lambda_{1}<\lambda_{2} \leq \cdots \leq \lambda_{n}$.

Moreover, it is known that the identity matrix $I$ has an $n$ multiple eigenvalue $\mu=1$ and $n$ independent eigenvectors

$$
\mathbf{v}^{1}=\left[\begin{array}{c}
1 \\
0 \\
\vdots \\
0
\end{array}\right], \mathbf{v}^{2}=\left[\begin{array}{c}
0 \\
1 \\
\vdots \\
0
\end{array}\right], \ldots, \mathbf{v}^{n}=\left[\begin{array}{c}
0 \\
0 \\
\vdots \\
1
\end{array}\right] .
$$

By matrix theory [41], the eigenvalues of $H \otimes I$ are $\lambda_{i} \mu=\lambda_{i}$ ( $n$ multiple for each $i$ ) and the corresponding eigenvectors are $\mathbf{u}^{i} \otimes \mathbf{v}^{j}$. Since $H \otimes I$ is clearly symmetric, the $n^{2}$ eigenvectors $\mathbf{u}^{i} \otimes \mathbf{v}^{j}$ are linearly independent. Therefore, if $\mathbf{e}^{T}(H \otimes I) \mathbf{e}=0$ then e must lie in the eigenspace of $H \otimes I$ spanned by eigenvectors $\mathbf{u} \otimes \mathbf{v}^{i}$ corresponding to the zero eigenvalue. Since

$$
\mathbf{u} \otimes \mathbf{v}^{i}=l\left[\begin{array}{c}
\mathbf{v}^{i} \\
\vdots \\
\mathbf{v}^{i}
\end{array}\right]
$$

we have

$$
\mathbf{e}=\sum_{i=1}^{n} k_{i} \mathbf{u} \otimes \mathbf{v}^{i}=l\left[\begin{array}{c}
\sum_{i=1}^{n} k_{i} \mathbf{v}^{i} \\
\vdots \\
\sum_{i=1}^{n} k_{i} \mathbf{v}^{i}
\end{array}\right]
$$

which implies that $\mathbf{e}^{1}=\mathbf{e}^{2}=\cdots=\mathbf{e}^{m}$. This occurs only when $\mathbf{e}^{1}=\mathbf{e}^{2}=\cdots=\mathbf{e}^{m}=0$. Hence, for any nontrivial solution $\mathbf{e}$, it must be in the subspace spanned by eigenvectors of $H \otimes I$ corresponding to the nonzero eigenvalues. Therefore, $\mathbf{e}^{T}(H \otimes I) \mathbf{e} \geq \lambda_{2}\|\mathbf{e}\|^{2}=2 \lambda_{2} V(\mathbf{e})$ and so,

$$
\dot{V}(\mathbf{e}) \leq-2 a \lambda_{2} V(\mathbf{e})+\sqrt{2} b\left\||W \|| V^{\frac{1}{2}}(\mathbf{e})<0\right.
$$

whenever $V(\mathbf{e})>\left[(b\||| W \mid\|) /\left(\sqrt{2} a \lambda_{2}\right)\right]^{2}$. Therefore, any solution of (1) outside the region $\Omega_{\rho}$ as defined in (6) will eventually enter into and remain in it.
Finally, we estimate the convergence time. For an arbitrary $\varepsilon>0$, let $\mathbf{e}$ be a solution of (9) with initial value $\mathbf{e}(0) \notin$ $\Omega_{\rho+\varepsilon}$ and $\tau>0$ be the time that $\mathbf{e}$ crosses the boundary of $\Omega_{\rho+\varepsilon}$, i.e., $\|\mathbf{e}(\tau)\|=\rho+\varepsilon$. Then it follows from the above that

$$
\dot{V}(\mathbf{e}) \leq-2 a \lambda_{2} V(\mathbf{e})+\sqrt{2} b\|\mid\| W \| V^{\frac{1}{2}}(\mathbf{e})
$$

for $t \in(0, \tau)$. Therefore, the solution of $V$ satisfies

$$
\left.\ln \left(2 a \lambda_{2} \sqrt{V(t)}-\sqrt{2} b|||W| \|\right)\right|_{0} ^{\tau} \leq-a \lambda_{2} \tau
$$

where $V(t)=V(\mathbf{e}(t))$. A straightforward manipulation yields the estimate (8). The proof is complete.

Remark 2. In contrast to the special case of isotropic swarm model considered in [19], the above discussion relies crucially on the eigenstructure of the interactive matrix $W$.

Notice that Theorem 2 only shows that the swarm members will aggregate and form a bounded cluster around the swarm center $\overline{\mathbf{x}}$, and $\rho$ provides a bound on the maximum ultimate swarm size. It does not, however, say anything about the motion of the members in the cluster. Next we show that for a class of attraction and repulsion functions satisfying Assumption 3, the swarm system (1) will converge to its equilibrium points. Hence the configuration of the swarm members tends to a constant arrangement. To state the result, we denote the set of equilibrium points of the swarm system (1) as

$$
\Omega_{e}=\{\mathbf{x}: \dot{\mathbf{x}}=\mathbf{0}\} .
$$

Definition 2: The swarm system (1) is completely stable if every solution converges to the equilibrium point set of the system.

Note that complete stability implies global convergence of a system to its equilibrium point set. It does not require the system to have unique equilibrium point. So it is a suitable concept for describing global dynamics of systems with multiple equilibria.

Now we are ready to present the following stability result.

Theorem 3: Under Assumptions 1-4, the swarm system (1) is completely stable.

Proof: First we note that under Assumption 3, the motion of individual $i$ is given by

$$
\dot{\mathbf{x}}^{i}=\sum_{j=1}^{m} w_{i j}\left[\nabla_{\mathbf{y}} p_{a}\left(\left\|\mathbf{x}^{i}-\mathbf{x}^{j}\right\|\right)-\nabla_{\mathbf{y}} p_{r}\left(\left\|\mathbf{x}^{i}-\mathbf{x}^{j}\right\|\right)\right] .
$$

Now we choose the Lyapunov function

$$
V(\mathbf{x})=\sum_{i=1}^{m-1} \sum_{j=i+1}^{m} w_{i j}\left[p_{a}\left(\left\|\mathbf{x}^{i}-\mathbf{x}^{j}\right\|\right)-p_{r}\left(\left\|\mathbf{x}^{i}-\mathbf{x}^{j}\right\|\right)\right] .
$$

Calculating the gradient of $V(\mathbf{x})$ with respect to $\mathbf{x}^{i}$ yields

$$
\begin{aligned}
\nabla_{\mathbf{x}^{i}} V(\mathbf{x}) & =\sum_{j=1}^{m} w_{i j}\left[\nabla p_{a}\left(\left\|\mathbf{x}^{i}-\mathbf{x}^{j}\right\|\right)-\nabla p_{r}\left(\left\|\mathbf{x}^{i}-\mathbf{x}^{j}\right\|\right)\right] \\
& =-\dot{\mathbf{x}}^{i}
\end{aligned}
$$


From this we can evaluate the time derivative of the Lyapunov function $V(\mathbf{x})$ along the motion of the system and obtain

$$
\dot{V}(\mathbf{x})=\sum_{i=1}^{m}\left[\nabla_{\mathbf{x}^{i}} V(\mathbf{x})\right]^{T} \dot{\mathbf{x}}^{i}=-\sum_{i=1}^{m}\left\|\dot{\mathbf{x}}^{i}\right\|^{2} \leq 0
$$

for all $t \geq 0$. Hence $V(\mathbf{x})$ decreases in $t$ unless $\dot{\mathbf{x}}^{i}=0$ for all $i$. To further conclude the complete stability of the system, we recall that by Theorem 2 every solution $\mathbf{x}(t)$ of (1) is bounded and eventually enters into the compact set $\Omega_{\rho}$ defined in (6). This implies that all the system solutions are bounded. Therefore, by LaSalle's Invariance Principle [42] we conclude that as $t \rightarrow \infty$ the solution $\mathbf{x}(t)$ converges to the largest positively invariant subset of the set defined by

$$
E=\{\mathbf{x}: \dot{V}(\mathbf{x})=0\} .
$$

It is clear from above discussion that $E=\Omega_{e}$ and is therefore positively invariant with respect to the system. Hence $\mathbf{x}(t) \rightarrow \Omega_{e}$ as $t \rightarrow \infty$.

Remark 3. It is clear from the proof that Theorem 3 will still hold if we assume the boundedness of solutions of the system instead of Assumption 2. The former is in general less stringent than the latter and can be fulfilled in most cases because of the long term attraction nature of the interaction in swarm members.

\section{NONRECIPROCAL SWARMS}

We now consider swarms that do not have the reciprocity property as specified before. In this case, the swarms may exhibit diverse behavior, from convergence to oscillation. We will present analytical and numerical simulation results.

\section{A. Aggregation and Stability Under the Detailed Balance Condition}

We first extend the preceding results to a class of nonreciprocal swarms whose coupling weights satisfying the "detailed balance condition" specified as below.

Assumption 5: The swarm described in (1) satisfies the detailed balance condition in weights, i.e., there exist certain scalars $\xi_{i}>0(i=1, \ldots, m)$ such that $\xi_{i} w_{i j}=\xi_{j} w_{j i}$ for all $i, j$.

Since we do not assume here the reciprocity of the swarms, the interactions between two individuals do not balance in general. Therefore, the swarm center as defined in the preceding section will not be stationary, but drifts with time. However, under Assumption 5, one can still obtain a simple characterization of the average motion of the swarm members by introducing the notion of "weighted center" defined as below instead of the "center" of the swarm.

Definition 3: The weighted center of the swarm members is defined as $\widetilde{\mathbf{x}}=\left(\sum_{i=1}^{m} \xi_{i} \mathbf{x}^{i}\right) /\left(\sum_{i=1}^{m} \xi_{i}\right)$.

Similar to Theorem 1, we have the following result concerning the average motion of the swarm members.

Theorem 4: Under Assumptions 1 and 5, the weighted center $\widetilde{\mathbf{x}}$ of the swarm described in (1) is stationary in $t$.
The proof is analogous to that of Theorem 1 by using the fact that $\xi_{i} w_{i j} \mathbf{f}\left(\mathbf{x}^{i}-\mathbf{x}^{j}\right)=-\xi_{j} w_{j i} \mathbf{f}\left(\mathbf{x}^{j}-\mathbf{x}^{i}\right)$ under the assumptions.

Next we show that the swarm members will move toward the weighted center and form a cohesive cluster around it.

Theorem 5: Under Assumptions 1, 2, and 5, all the members of the swarm described in (1) will eventually enter into and remain in the bounded region

$$
\Omega_{\sigma}=\left\{\mathbf{x}: \sum_{i=1}^{m} \xi_{i}\left\|\mathbf{x}^{i}-\widetilde{\mathbf{x}}\right\|^{2} \leq \sigma^{2}\right\}
$$

where $\sigma=\left(b \xi_{\max }\||L W|\|\right) /\left(a \gamma_{2}\right)$ with $L=$ $\operatorname{diag}\left[\sqrt{\xi_{1}}, \ldots, \sqrt{\xi_{m}}\right], \quad\|L W\|=\sum_{i, j=1}^{n} \sqrt{\xi_{1}} w_{i j}$, $\xi_{\max }=\max _{1 \leq i \leq m}\left\{\xi_{i}\right\}>0$, and $\gamma_{2}$ the second smallest real eigenvalue of the symmetric matrix $K=\left[k_{i j}\right]$ defined by

$$
k_{i j}=\left\{\begin{array}{l}
-\xi_{i} w_{i j}, i \neq j \\
\sum_{l=1, l \neq i}^{m} \xi_{i} w_{i l}, i=j
\end{array} .\right.
$$

Moreover, for an arbitrary small $\varepsilon>0$, the convergence of the swarm to $\Omega_{\sigma+\varepsilon}$ (i.e., the $\varepsilon$-neighborhood of $\Omega_{\sigma}$, defined as in (6) with radius $\sigma+\varepsilon$ ) from an initial state $\mathbf{x}(0)$ outside the set will occur in finite time bounded by

$$
\tau \leq \frac{\xi_{\max }}{a \gamma_{2}} \ln \left(\frac{\sigma_{0}-\sigma}{\varepsilon}\right)
$$

where $\sigma_{0}=\sqrt{\sum_{i=1}^{m} \xi_{i}\left\|\mathbf{x}^{i}(0)-\widetilde{\mathbf{x}}\right\|^{2}}>\sigma+\varepsilon$.

Proof: Let $\mathbf{d}^{i}=\mathbf{x}^{i}-\widetilde{\mathbf{x}}$. By definition of the weighted center $\widetilde{\mathbf{x}}$, we have

$$
\dot{\mathbf{d}}^{i}=-\sum_{j=1}^{m} w_{i j}\left(\mathbf{d}^{i}-\mathbf{d}^{j}\right)\left[f_{a}\left(\left\|\mathbf{d}^{i}-\mathbf{d}^{j}\right\|\right)-f_{r}\left(\left\|\mathbf{d}^{i}-\mathbf{d}^{j}\right\|\right)\right] .
$$

To estimate $\mathbf{d}^{i}$, we take the Lyapunov function for (12) as $V(\mathbf{d})=\sum_{i=1}^{m} V_{i}\left(\mathbf{d}^{i}\right)$ where $\mathbf{d}=\left[\mathbf{d}^{1 T}, \ldots, \mathbf{d}^{m T}\right]^{T}$ and

$$
V_{i}\left(\mathbf{d}^{i}\right)=\frac{1}{2} \xi_{i} \mathbf{d}^{i T} \mathbf{d}^{i}
$$

Similar to the proof of Theorem 2 , by making use of the assumptions and the fact that $\|\mathbf{d}\|^{2} \geq\left(2 / \xi_{\max }\right) V(\mathbf{d})$ and $\left\|\mathbf{d}^{i}\right\| \leq \sqrt{\left(2 / \xi_{i}\right) V(\mathbf{d})}$ for all $i$, we can evaluate the derivative of $V(\mathbf{d})$ along solutions of the system and obtain

$$
\begin{aligned}
\dot{V}(\mathbf{d})= & -\sum_{i=1}^{m} \sum_{j=1}^{m} \xi_{i} w_{i j} \mathbf{d}^{i T}\left(\mathbf{d}^{i}-\mathbf{d}^{j}\right) \\
& \times\left[f_{a}\left(\left\|\mathbf{d}^{i}-\mathbf{d}^{j}\right\|\right)-f_{r}\left(\left\|\mathbf{d}^{i}-\mathbf{d}^{j}\right\|\right)\right] \\
\leq & -a \sum_{i=1}^{m} \sum_{j=1}^{m} \xi_{i} w_{i j} \mathbf{d}^{i T}\left(\mathbf{d}^{i}-\mathbf{d}^{j}\right)+b \sum_{i=1}^{m} \sum_{j=1}^{m} \xi_{i} w_{i j}\left\|\mathbf{d}^{i}\right\| \\
\leq & -a \mathbf{d}^{T}(K \otimes I) \mathbf{d}+\sqrt{2} b\|\mid\| L W \| V^{\frac{1}{2}}(\mathbf{d}) \\
\leq & -2 a \gamma_{2} \xi_{\max }^{-1} V(\mathbf{d})+\sqrt{2} b\|L W\| V^{\frac{1}{2}}(\mathbf{d}) \\
< & 0
\end{aligned}
$$

whenever $V(\mathbf{d})>\left[\left(b \xi_{\max }|||L W|||\right) /\left(\sqrt{2} a \gamma_{2}\right)\right]^{2}$. Therefore, any solution of (1) outside the region $\Omega_{\sigma}$ as defined in (10) will eventually enter into and remain in it. Similar to 
the proof of Theorem 2, we can further estimate the bound of convergence time and obtain (11).

Furthermore, we can also prove the complete stability of the nonreciprocal swarm under the detailed balance condition.

Theorem 6: Under Assumptions 1-3, and 5, the swarm described in (1) is completely stable.

Proof: With Assumption 3, we can take the Lyapunov function candidate

$$
V(\mathbf{x})=\sum_{i=1}^{m-1} \sum_{j=i+1}^{m} \xi_{i} w_{i j}\left[p_{a}\left(\left\|\mathbf{x}^{i}-\mathbf{x}^{j}\right\|\right)-p_{r}\left(\left\|\mathbf{x}^{i}-\mathbf{x}^{j}\right\|\right)\right]
$$

The gradient of $V(\mathbf{x})$ with respect to $\mathbf{x}^{i}$ is given by

$$
\begin{aligned}
\nabla_{\mathbf{x}^{i}} V(\mathbf{x}) & =\sum_{j=1}^{m} \xi_{i} w_{i j}\left[\nabla p_{a}\left(\left\|\mathbf{x}^{i}-\mathbf{x}^{j}\right\|\right)-\nabla p_{r}\left(\left\|\mathbf{x}^{i}-\mathbf{x}^{j}\right\|\right)\right] \\
& =-\xi_{i} \dot{\mathbf{x}}^{i}
\end{aligned}
$$

Therefore, along the motion of the system we have

$$
\dot{V}(\mathbf{x})=\sum_{i=1}^{m}\left[\nabla_{\mathbf{x}^{i}} V(\mathbf{x})\right]^{T} \dot{\mathbf{x}}^{i}=-\sum_{i=1}^{m} \xi_{i}\left\|\dot{\mathbf{x}}^{i}\right\|^{2} \leq 0
$$

for all $t \geq 0$. Hence, by the boundedness of solutions of the system as specified in Theorem 5 and using LaSalle's invariant principle [42], we have $\mathbf{x}(t) \rightarrow \Omega_{e}$ as $t \rightarrow \infty$.

\section{B. Oscillatory Motion in Nonreciprocal Swarms}

For a general nonreciprocal swarm model in (1) without the detailed balance condition, it is usually difficult to analytically investigate collective behavior of the swarm members. To get some ideas about the motion of nonreciprocal swarms, we have performed some numerical simulations for the swarm model described by (1) and (5). It is observed that in certain cases, the swarms eventually evolve into self-organized oscillatory motion.

Figs. 1-3 show the results in one of our simulations, where the attraction-repulsion function is taken in the form of (5) with parameters $m=10, a=1, b=20$, and $c=0.2$. The coupling matrix $W$ is generated randomly and scaled so that $w_{i i}=0,0<w_{i j}<1$ for all $i \neq j$. Due to its large size, we do not include here the value of $W$ explicitly. It is available upon request. We run the simulation for 3000 seconds.

It can be seen from Fig. 1 that at the beginning phase of the simulation, the swarm members gradually aggregate and form a cohesive cluster. Then, they continuously move in the the same direction as a group while keeping mutual spacing, and eventually evolve into an expanding spiral motion as time increases. Fig. 2 illustrates the path of the swarm center. Fig. 3 is a magnification of a portion of the center trajectory, which evolves on the curves from left to right as time increases.

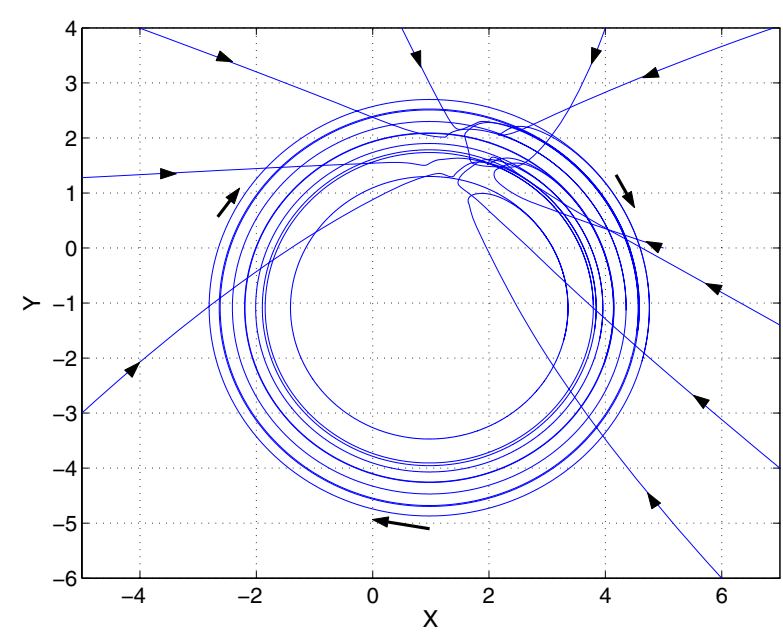

Fig. 1. Swarm member trajectories.

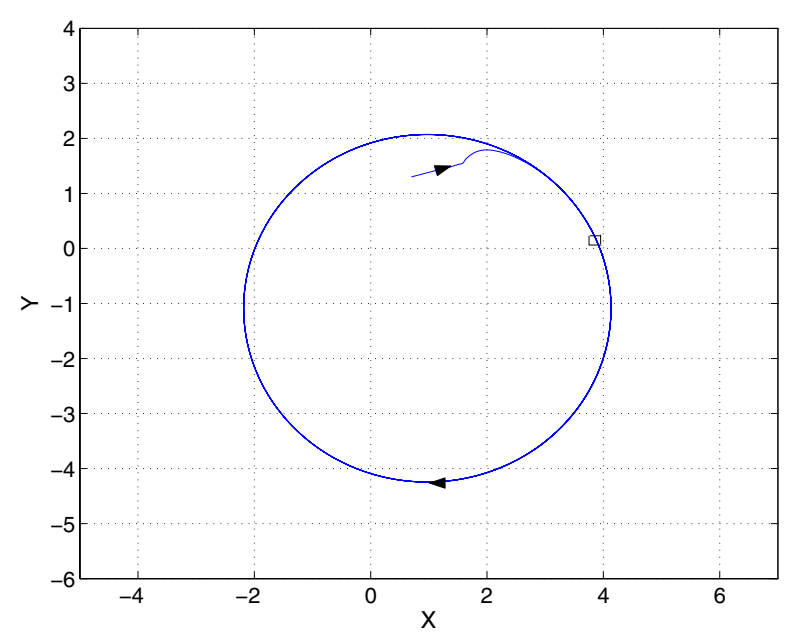

Fig. 2. Swarm center trajectory.

Observe that such an oscillation is merely due to the non-symmetry of coupling weights among swarm members, having nothing to do with any external effects. This together with the previous aggregation and stability properties shows explicitly the effect of the coupling pattern on the swarm dynamics.

\section{COnClusions}

We have analyzed a class of anisotropic swarms and shown aggregation behavior and complete stability of the swarms under reciprocal weight condition and detailed balance condition, respectively. The anisotropic connectivity allows for the consideration of non-identical interaction ability or efficiency among different swarm individuals. The results obtained here generalize a recent work [19] on isotropic swarm model and provide further insight into the effect of the interaction pattern on self-organized motion in a swarm. 


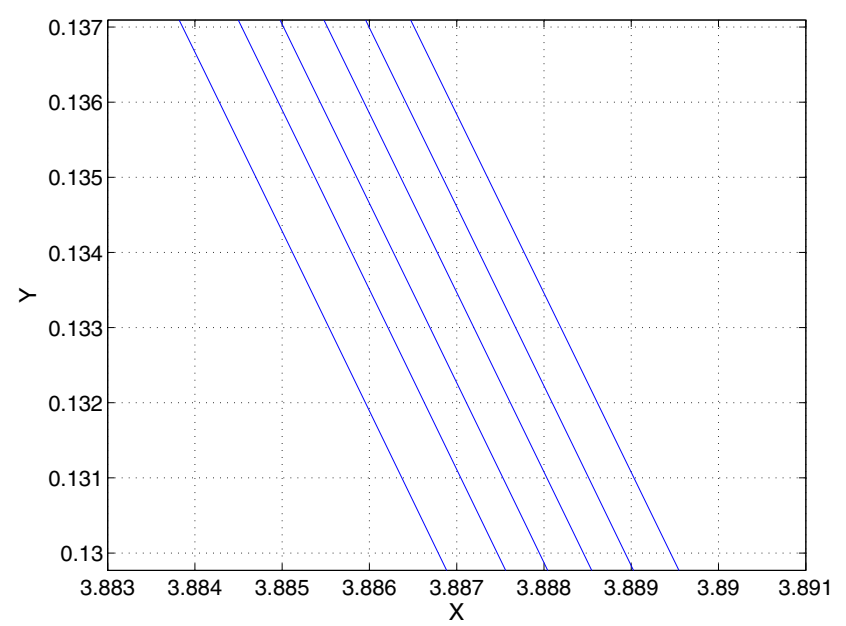

Fig. 3. A magnification of the center trajectory in the small rectangle shown in Fig. 2.

\section{REFERENCES}

[1] C. M. Breder, "Equations descriptive of fish schools and other animal aggregations," Ecology, vol. 35, no. 3, pp. 361-370, 1954.

[2] J. Bender and R. Fenton, "On the flow capacity of automated highways," Transport. Sci., vol. 4, pp. 52-63, Feb. 1970.

[3] F. Giulietti, L. Pollini, and M Innocenti, "Autonomous formation flight,” IEEE Control Systems Magazine, vol. 20, pp. 34-44, Dec. 2000 .

[4] T. Balch and R. C. Arkin, "Behavior-based formation control for multirobot teams," IEEE Trans. Robot Automat., vol. 14, pp. 926939, Dec. 1998.

[5] J. P. Desai, J. Ostrowski, and V. Kumar, "Controlling formations of multiple mobile robots," in Proc. IEEE Int. Conf. Robotics Automation, Leuven, Belgium, pp. 2864-2869, May 1998.

[6] M. Egerstedt and X. Hu, "Formation constrained multi-agent control ," IEEE Trans. Robot. Automat., vol. 17, pp. 947-951, Dec. 2001.

[7] P. Ögren, M. Egerstedt, and X. Hu, "A control Lyapunov function approach to multiagent coordination," IEEE Trans. Robot. Automat., vol. 18 , no. 5, pp. 847-851, Oct. 2002.

[8] N. E. Leonard and E. Fiorelli, "Virtual leaders, artificial potentials and coordinated control of groups," in Proc. IEEE Conf. Decision Control, Orlando, FL, pp. 2968-2973, Dec. 2001.

[9] P. Tabuada, G. J. Pappas, and P. Lima, "Feasable formations of multiagent systems," in Proc. Amer. Control Conf., Arlington, VA, pp. 56-61, June 2001.

[10] J. H. Reif and H. Wang, "Social potential fields: A distributed behavioral control for autonomous robots," Robot. Auton. Syst., vol. 27, pp. 171-194, 1999.

[11] I. Suzuki and M, Yamashita, "Distributed anonymous mobile robots: Formation of geometric patterns," SIAM J. Comput., vol. 28, no. 4, pp. 1347-1363, 1999.

[12] K. Jin, P. Liang, and G. Beni, "Stability of synchronized distributed control of discrete swarm structures," in Proc. IEEE Int. Conf. Robotics Automation, San Diego, CA, pp. 1033-1038, May 1994.

[13] G. Beni and P. Liang, "Pattern reconfiguration in swarmsconvergence of a distributed asynchronous and bounded iterative algorithm," IEEE Trans. Robot Automat., vol. 12, pp. 485-490, June 1996.

[14] Y. Liu, K. M. Passino, and M. M. Polycarpou, "Stability analysis of M-dimensional asynchronous swarms with a fixed communication topology," IEEE Trans. Automat. Contr, vol. 48, pp. 76-95, Jan. 2003.

[15] Y. Liu, K. M. Passino, and M. Polycarpou, "Stability analysis of onedimensional asynchronous mobile swarms," IEEE Trans. Automat. Contr., vol. 48, pp. 1848-1854, Oct. 2003.

[16] D. Swaroop, J. K. Hedrick, C. C. Chien, and P. Ioannou, "A comparison of spacing and headway control laws for automatically controlled vehicles," Veh. Syst. Dyna., vol. 23, pp. 597-625, 1994.
[17] S. Darbha and K. P. Rajagopal, "Intelligent cruise control systems and traffic flow stability," Transport. Res. C, vol. 7, pp. 329-352, 1999.

[18] H. G. Tanner, A. Jadbabaie, and G. J. Pappas, "Stable flocking of mobile agents, Part I: Fixed topology; Part II: Dynamic topology," in Proc. 42nd IEEE Conf. Decision Contr., Maui, Hawaii USA, pp. 2010-2015; pp. 2016-2021, Dec. 2003.

[19] V. Gazi and K. M. Passino, "Stability analysis of swarms," IEEE Trans. Automat. Contr., vol. 48, pp. 692-697, Apr. 2003.

[20] V. Gazi and K. M. Passino, "Stability analysis of social foraging swarms," IEEE Trans. Syst., Man, Cybern., Part B: Cybern., vol. 34, no. 1, pp. 539-557, Feb. 2004.

[21] A. Jadbabaie, J. Lin, and A. S. Morse, "Coordination of groups of mobile autonomous agents using nearest neighbor rules," IEEE Trans. Automat. Contr., vol. 48, pp. 988-1001, June 2003.

[22] R. Sepulchre, D. Paley, and N. Leonard, "Collective motion and oscillator synchronization," in Proc. 2003 Block Island Workshop on Cooperative Control, V. J. Kumar, N. E. Leonard, and A. S. Morse, eds., Springer-Verlag, Berlin, 2003.

[23] T. Chu, L. Wang, and T. Chen, "Self-organized motion in anisotropic swarms," J. Contr. Theory Appl., vol. 1, no. 1, pp. 77-81, 2003.

[24] T. Chu, L. Wang, and S. Mu, "Collective behavior analysis of an anisotropic swarm model," in Proc. 16th Inter. Symp. Math. Theory Networks Syst. (MTNS 2004, Paper ID: REG-345), Leuven, Belgium, July 2004.

[25] L. Wang, H. Shi, T. Chu, W. Zhang, and L. Zhang, "Aggregation of forging swarms," in Advances in Artificial Intelligence, pp. 766-777, Lecture Notes in Artificial Intelligence, vol. 3339, Springer-Verlag, 2004.

[26] H. Shi, L. Wang, and T. Chu, "Swarming behavior of multi-agent systems," J. Contr. Theory Appl., vol. 2, Dec. 2004.

[27] B. Liu, T. Chu, L. Wang., and Z. Wang, "Swarm dynamics of a group of mobile autonomous agents," Chinese Phys. Lett., vol. 22, no. 1, pp. 254-257, 2005.

[28] S. Mu, T. Chu, and L. Wang, "Coordinated collective motion in a motile particle group with a leader," Physica A, in press, 2005.

[29] W. Wang and J.-J. E. Slotine, "On partial contraction analysis for coupled nonlinear oscillators," Biolo. Cybern., vol. 92, n. 1, pp. 3853, Jan. 2005.

[30] E. Justh and P. Krishnaprasad, "Equilibria and steering laws for planar formations," Syst. Contr. Lett., vol. 52, pp. 25-38, 2004.

[31] Z. Lin, M. Broucke, and B. Francis, "Local control strategies for groups of mobile autonomous agents," IEEE Trans. Automat. Contr., vol. 49, pp. 622-629, April 2004.

[32] A. V. Savkin, "Coordinated collective motion of groups of autonomous mobile robots: analysis of Vicsek's model," IEEE Trans. Automat. Contr., vol. 49, pp. 981-983, June 2004.

[33] J. A. Fax and R. M. Murray, "Information flow and cooperative control of vehicle formations," IEEE Trans. Automat. Contr, vol. 49, pp. 1465-1476, Sept. 2004.

[34] R. Olfati-Saber and R. M. Murray, "Consensus problems in networks of agents with switching topology and time-delays," IEEE Trans. Automat. Contr., vol. 49, pp. 1520-1533, Sept. 2004.

[35] K. Warburton and J. Lazarus, "Tendency-distance models of social cohesion in animal groups," J. Theoret. Biolo., vol. 150, pp. 473-488, 1991

[36] A. Okubo, "Dynamical aspects of animal grouping: swarms, schools, flocks, and herds," Adv. Biophys., vol. 22, pp. 1-94, 1986.

[37] D. Grünbaum and A. Okubo, "Modeling social animal aggregations ," Frontiers in Theoretical Biology, Springer-Verlag, New York, vol. 100, Lecture Notes in Biomathematics, pp. 296-325, 1994.

[38] T. Vicsek, A. Czirok, E. Ben-Jacob, I. Cohen, and O. Shochet, "Novel type of phase transition in a system of self-driven particles," Phys. Rev. Lett., vol. 675, no. 6, pp. 1226-1229, Aug. 1995.

[39] N. Shimoyama, K. Sugawa, T. Mizuguchi, Y. Hayakawa, and M. Sano, "Collective motion in a system of motile elements," Phys. Rev. Lett., vol. 76, no. 20, pp. 3870-3873, May 1996.

[40] H. Levine and W. J. Rappel, "Self-organization in systems of selfpropelled particles," Phys. Rev. E, vol. 63, no. 1, pp. 017101-1017101-4, Jan. 2001.

[41] R. A. Horn, C. R. Johnson, Matrix Analysis, Cambridge University Press, Cambridge, 1990.

[42] H. Khalil, Nonlinear Systems, 3rd ed., Upper Saddle River, NJ: Prentice-Hall, 1999. 